\title{
Genome-wide analysis of DNA methylation and gene expression defines molecular characteristics of Crohn's disease-associated fibrosis
}

Tammy Sadler ${ }^{1 \dagger}$, Jeffrey M. Bhasin ${ }^{2,3+}$, Yaomin Xu' ${ }^{4}$, Jill Barnholz-Sloan ${ }^{5}$, Yanwen Chen ${ }^{5}$, Angela H. Ting ${ }^{2,3^{*}}$ and Eleni Stylianou ${ }^{1,6^{*}}$

\begin{abstract}
Background: Fibrosis of the intestine is a common and poorly understood complication of Crohn's disease (CD) characterized by excessive deposition of extracellular matrix and accompanied by narrowing and obstruction of the gut lumen. Defining the molecular characteristics of this fibrotic disorder is a vital step in the development of specific prediction, prevention, and treatment strategies. Previous epigenetic studies indicate that alterations in DNA methylation could explain the mechanism by which mesenchymal cells adopt the requisite pro-fibrotic phenotype that promotes fibrosis progression. However, to date, genome-wide analysis of the DNA methylome of any type of human fibrosis is lacking. We employed an unbiased approach using deep sequencing to define the DNA methylome and transcriptome of purified fibrotic human intestinal fibroblasts (HIF) from the colons of patients with fibrostenotic CD.

Results: When compared with normal fibroblasts, we found that the majority of differential DNA methylation was within introns and intergenic regions and not associated with CpG islands. Only a low percentage occurred in the promoters and exons of genes. Integration of the DNA methylome and transcriptome identified regions in three genes that inversely correlated with gene expression: wingless-type mouse mammary tumor virus integration site family, member 2B (WNT2B) and two eicosanoid synthesis pathway enzymes (prostacyclin synthase and prostaglandin D2 synthase). These findings were independently validated by RT-PCR and bisulfite sequencing. Network analysis of the data also identified candidate molecular interactions relevant to fibrosis pathology.

Conclusions: Our definition of a genome-wide fibrosis-specific DNA methylome provides new gene networks and epigenetic states by which to understand mechanisms of pathological gene expression that lead to fibrosis. Our data also provide a basis for development of new fibrosis-specific therapies, as genes dysregulated in fibrotic Crohn's disease, following functional validation, can serve as new therapeutic targets.
\end{abstract}

Keywords: DNA methylome, Transcriptome, Intestinal fibrosis, Next generation sequencing, RNA seq, Omics, Crohn's disease, Inflammatory bowel disease

\footnotetext{
* Correspondence: tinga@ccf.org; eleni.stylianou@att.net

${ }^{\dagger}$ Equal contributors

${ }^{2}$ Department of Molecular Medicine, Cleveland Clinic Lerner College of

Medicine at Case Western Reserve University, Cleveland, OH, USA

${ }^{1}$ Department of Pathobiology, Cleveland Clinic Lerner Research Institute,

9500 Euclid Avenue/NC-22, Cleveland, $\mathrm{OH}$ 44195, USA

Full list of author information is available at the end of the article
} 


\section{Background}

Intestinal fibrosis is a devastating complication of Crohn's disease (CD), a major type of inflammatory bowel disease (IBD) [1]. Characterized by a chronic transmural inflammation of the intestine, CD is disabling, incurable, and of unknown etiology. The associated fibrosis comprises prolonged abnormal wound repair and tissue remodeling leading to excessive deposition of a collagen-containing extracellular matrix (ECM). Hypertrophy of the submucosa and muscularis is a major contributor to the increased rigidity and thickness of the bowel wall. These changes typically lead to stricture formation and fibro-stenosis, a major cause of serious complications and surgical procedures in $C D$ patients. In the absence of fibrosis-specific drugs, antiinflammatory therapies have not prevented or reduced the incidence of fibrosis. For the $C D$ patients that succumb to this complication, surgical intestinal resection is currently the only treatment option and provides temporary symptomatic relief without cure or alteration of disease progression [2]. In this context, the ability to predict the patients that develop fibrosis remains an important challenge that would significantly improve the clinical management of IBD.

A number of factors have been proposed to have a role in the etiology of CD $[1,3,4]$. These include genetic susceptibility, defects in innate immunity, undefined environmental factors, and alterations in the microbiome. Recent genome-wide association studies appear to explain only a minority of the risk associated with development of $C D[5,6]$. The high rate of discordance among monozygotic twins and the increased prevalence of CD over recent decades suggest environmental factors may be at play. As epigenetic changes are dynamically responsive to the environment, they are likely to play a key role in the pathogenesis of fibrosis and to offer a molecular explanation for how the intestine becomes pro-fibrotic.

DNA methylation is the addition of a methyl group to the 5-position of the DNA base cytosine. Genome-wide changes in DNA methylation have been shown to be major contributors to cancer, mammalian development, gene transcription, and phenotype in a range of diseases $[7,8]$, including a variety of autoimmune and inflammatory conditions. Recent epigenetic profiling of IBD patients has comprised DNA methylome signatures of colon tissue, whole blood, and B cells for both major types of inflammatory bowel disease: ulcerative colitis (UC) and CD [9-13]. Of the few previous studies, two of these correlated changes in DNA methylation with gene expression $[12,13]$ and only one analyzed a purified (B cells) cell type [10]. Furthermore, of the three previous studies that have analyzed epigenetic changes in intestinal fibrogenesis, none of these defined changes in DNA methylation. One, from our lab, showed that chromatin modifications are linked with activation of type I collagen gene expression in endothelial to mesenchymal transition [14], a feature of intestinal fibrosis in vivo. The two other labs focused on specific miRNAs in the fibrotic intestine $[15,16]$. Moreover, all published studies to date of the DNA methylation in fibrotic diseases have been limited to studying restricted subsets of genes or to the use of microarrays that lack genome-wide coverage [17-23]. We report here the use of an unbiased, genome-wide approach to define the DNA methylome and the transcriptome of fibroblasts isolated from colons of control and CD patients. This approach avoids the issues of heterogeneity of tissue/biopsy samples and employs next generation sequencing using the methyl-CpG binding domain (MBD) of MBD2, called MBD-isolated genome sequencing (MiGS). MiGS is based on the capacity of MBD2 to bind with high affinity and specificity to DNA containing densely methylated cytosines [24]. A sequencing library heavily enriched for these methylated sequences within sheared genomic DNA is coupled to next generation sequencing so that the location of DNA methylation at specific genomic loci can be quantified and compared. We integrated this information with RNA sequencing (RNA-seq) data to identify key molecular interactions that lead to fibrosis pathology. Our identification of key differentially methylated regions (DMRs) in intestinal fibrosis provides new molecular characteristics for fibrostenotic CD and a resource for studying epigenetic mechanisms that could help classify different stages of fibrosis and identify patients predisposed to developing this major complication of IBD.

\section{Results}

Genome-wide changes in DNA methylation in fibrotic human intestinal fibroblasts

Genome-wide DNA methylation profiles were generated from human intestinal fibroblasts (HIF) isolated from colon resection specimens that were either normal or from patients with fibrostenotic CD. We identified statistically significant regions of differential DNA methylation between the two groups at a false discovery rate (FDR) threshold of $<5 \%$. Both quantitative and qualitative differences in DNA methylation were detected (Additional file 1). Qualitative differences were defined as sharp yes/no DNA methylation with a clear presence or absence of DNA methylation between the normal and fibrotic groups. In sharp yes/no DMRs, one group has zero or statistically near-zero sequencing reads, indicating a lack of methylation, whereas the other group shows a strong enrichment of reads, indicating the presence of methylation. Heatmap visualization and hierarchical clustering of the sharp yes/no DMRs showed a striking difference between the DMRs in normal and CD samples (Fig. 1a). These sharp yes/no DMRs were 


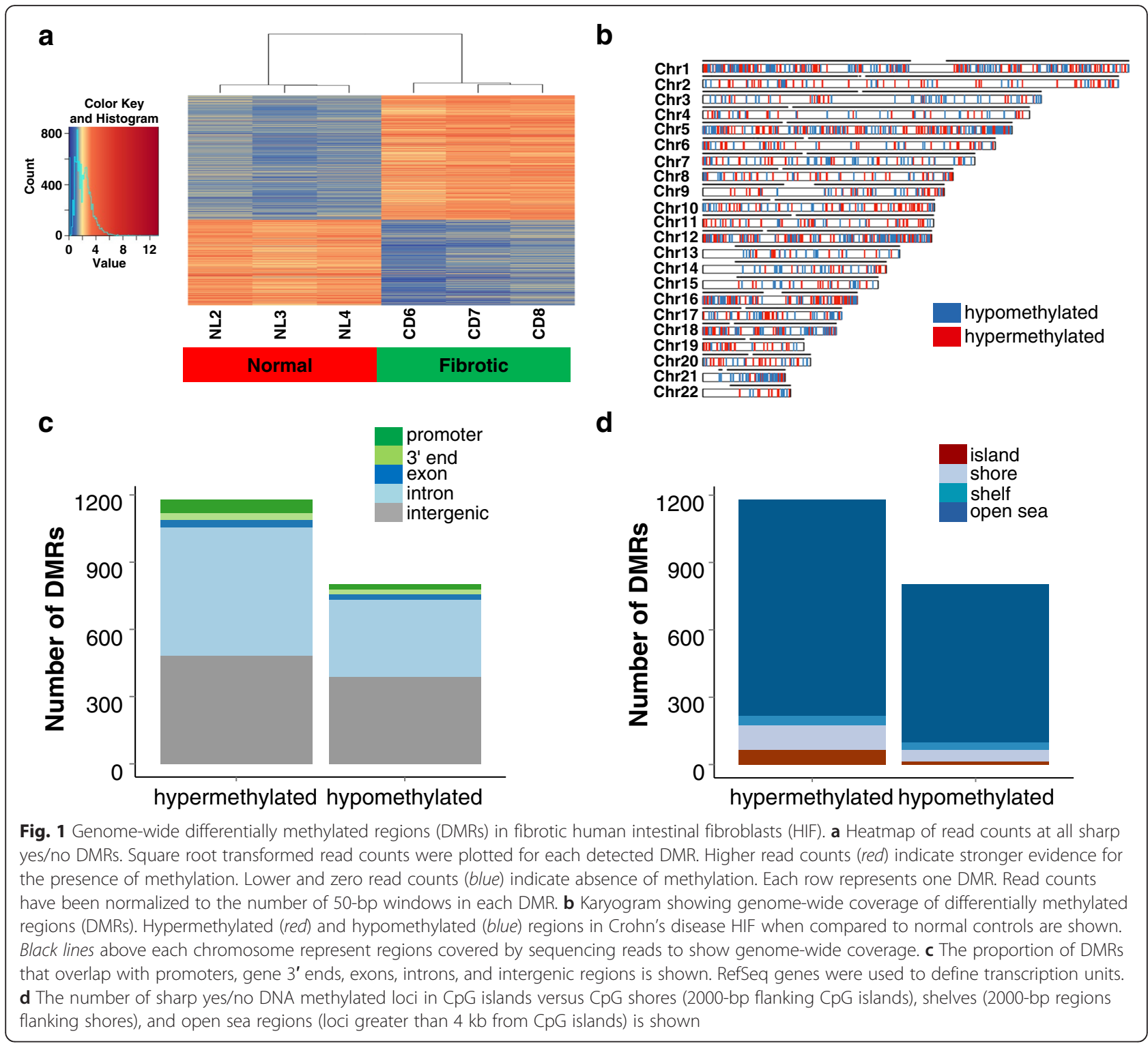

detected throughout the genome on all 22 autosomes (Fig. 1b). Of the sharp yes/no DMRs, 1180 DMRs represent hypermethylation in the fibrotic samples as compared to the normal samples, and 802 represent hypomethylation. We focused further analysis on qualitative differences in methylation because the quantitative differences (where both conditions are statistically different and have high numbers of reads) are likely methylated in all conditions [25].

\section{Genomic and CpG island context of differential DNA methylation in intestinal fibrosis}

Evidence from recent studies suggests that the genomic location of DNA methylation is a major contributor to the type of function performed by this epigenetic modification $[7,8]$. Based on the global distribution of the hyper- and
hypo-DNA methylated loci, we determined the frequency of loci in promoter, inter-, and intragenic regions (Fig. 1c and Additional file 1). Promoter regions were defined as $+1000 \mathrm{bp}$ to -500 bp relative to transcription start sites, 3 ' end gene regions as $+1000 \mathrm{bp}$ to $-1000 \mathrm{bp}$ of transcription termination sites, and intergenic regions as the remaining regions of the genome. The NCBI reference sequence database (RefSeq) was used to define the transcription units for all genes. In the cases where DMRs were located in multiple gene regions, the overlaps were prioritized as follows: promoter $>3^{\prime}$ end $>$ exon $>$ intron $>$ intergenic. Only a minor percentage of the mapped differential DNA methylation occurred in promoters $(5.0$ and $2.7 \%$ for hypermethylated and hypomethylated DMRs, respectively), and a large percentage occurred in intergenic 
regions (40.8 and $48.4 \%$ ). DNA methylation within gene bodies has also been reported to have an important role in transcriptional control [26]. While only a small percentage of DMRs overlapped with annotated exons (3.0 and $2.9 \%)$, DMRs were abundant within introns (48.6 and $43.1 \%$ ).

We also asked whether methylated promoter $\mathrm{CpG}$ islands (CGIs), associated with repression of gene transcription [27], are a feature of intestinal fibrosis (Fig. 1d). The vast majority of differential DNA methylation was found outside CGIs. For hypermethylated and hypomethylated DMRs, respectively, only 5.9 and $1.9 \%$ were in CGIs, 9.7 and $6.4 \%$ in shores (2000-bp flanking CpG islands), and 4.0 and $4.1 \%$ in shelves (2000-bp flanking shore regions). Of all the DMRs, the open sea regions (loci greater than $4 \mathrm{~kb}$ from $\mathrm{CpG}$ islands) contained most of the differential non-CpG island methylation (86.3 and $87.7 \%$ \%). Published data indicate that alterations in DNA methylation outside CGIs in shelves/ shores can play a role in gene transcription and may be cell-type specific [28].

\section{Genome-wide transcriptome analysis of fibrotic and normal intestinal fibroblasts}

RNA-seq analysis identified the fibrosis-associated changes in the HIF transcriptome associated with changes in DNA methylation. Using established criteria for analyzing RNA-seq data from normal and fibrotic RNA, we found 72 genes that were differentially expressed (Benjamin-Hochberg adjusted $p$ value $<0.05, \mathrm{FDR}<5 \%$, Fig. $2 \mathrm{a}$ and Additional file 2). The consistency of gene expression within each group is evident from the hierarchical clustering dendrogram. Of the 72 differentially expressed genes, 31 were downregulated and 41 upregulated. Only three genes, toll-like receptor 4 (TLR4) [29], interleukin 33, (IL-33) [30], and insulin-like growth factor 1 (IGF-1)

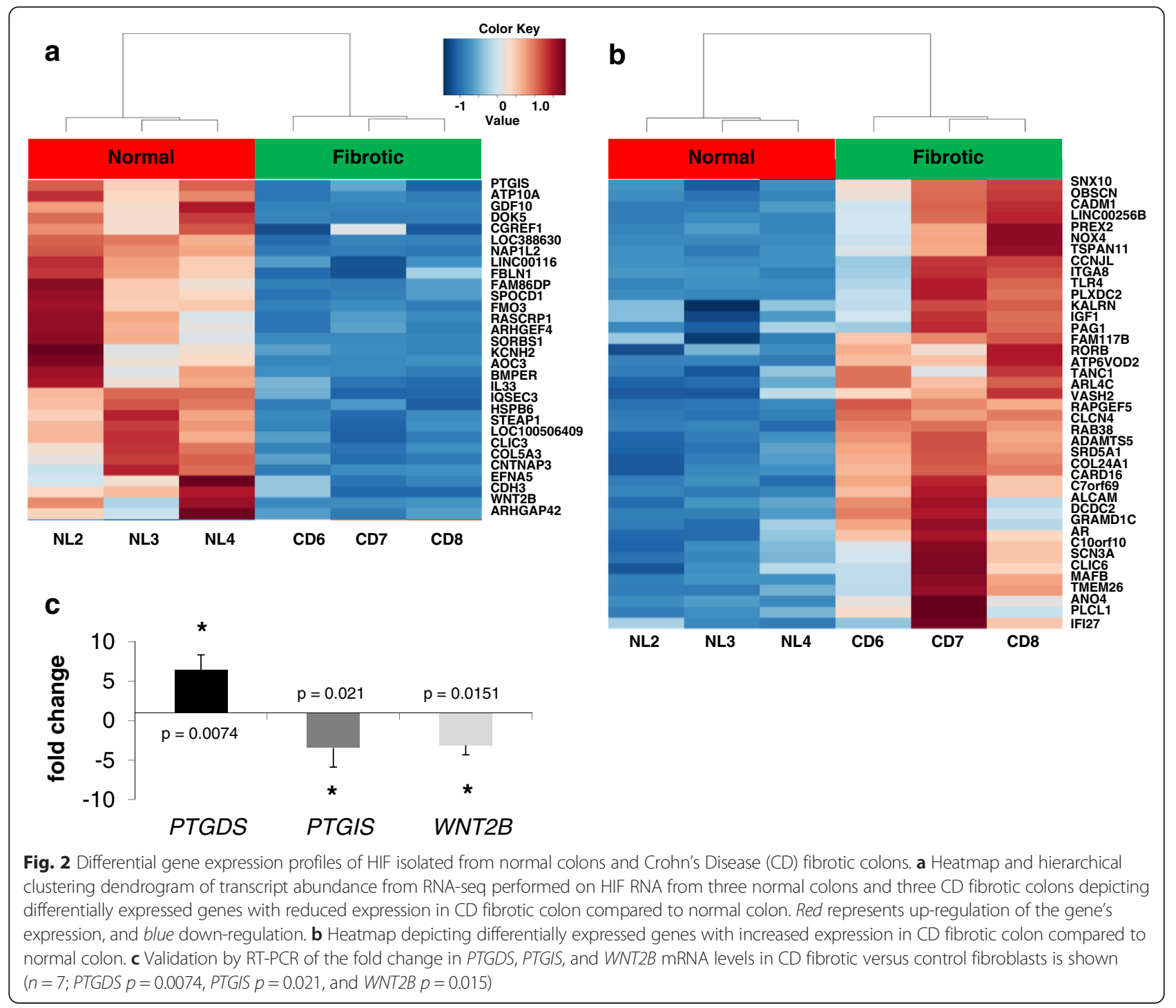


[31], have been previously reported or hypothesized to have functions in intestinal inflammation, IBD, or other fibrotic diseases [32, 33].

\section{WNT2B, PTGIS, and PTGDS are subject to differential expression and differential DNA methylation}

We next examined which of the sharp yes/no DMRs in promoter regions that overlapped with genes were differentially expressed (Table 1). Promoter hypermethylation of two protein-coding genes, wingless-type mouse mammary tumor virus integration site family, member $2 \mathrm{~B}$ (WNT2B) [34] and prostacyclin synthase (PTGIS) [35], were inversely correlated with alterations in their mRNA levels (Table 1). Validation by RT-PCR showed that the changes in PTGIS mRNA and WNT2B mRNA were decreased in fibrotic compared with normal HIF (Fig. 2b), consistent with the observed hypermethylation of each gene. In contrast, the mRNA level of prostaglandin D2 synthase (PTGDS), another member of the eicosanoid (prostaglandin) family [36], was increased in fibrotic HIF (Fig. 2b). Bisulfite sequencing was performed on the WNT2B, PTGIS, and PTGDS genes (Figs. 3a, b and Additional file 3) to validate the DMRs at base-pair resolution. The DMRs in the $W N T 2 B$ and PTGIS promoters were confirmed to be hypermethylated (Fig. 3a, b). Bisulfite sequencing confirmed that PTGDS was hypomethylated in a 550-bp region spanning its promoter and coding sequence (Additional file 3). These findings corroborate the corresponding changes in expression and DNA methylation levels for all three genes (Additional file 3, Figs. 2b and 3a, b).

\section{Novel functional gene networks in Crohn's disease- associated fibrosis}

As a first step in determining how differential DNA methylation contributes to intestinal fibrosis pathogenesis, we used the GeneMANIA algorithm to predict network-based functional associations between genes differentially methylated and differentially expressed in fibrotic HIF (list of input genes in Additional file 4). The attributed functions fall into two main groups. The first is extracellular matrix structure and organization, which is highly relevant to fibrosis of the intestine and other fibrotic disorders [37, 38]. The second is guanine nucleotide exchange factor signaling, including the small GTP proteins RHO and RAC [39], previously described in gastrointestinal ulcer healing and in other fibrotic diseases but not in intestinal fibrosis [40, 41].

Sub-networks that include the three differentially methylated and expressed genes WNT2B, PTGIS, and PTGDS (Fig. 4) and the genes that interact with them were identified (Additional files 5, 6, and 7). PTGDS and PTGIS were associated with fibulin 1 (FBLN1) and IGF-1, both linked with extracellular matrix structure and fibrosis [31, 42]. PTGIS is also linked with other genes involved in the organization and structure of the extracellular matrix, for example, ADAM metalloprotease with thrombospondin motifs 5 (ADAMTS5) and the alpha 2 chain of type I collagen (COL1A2) [42-44]. There are also other interactions not previously described in any fibrotic disease; for example, both $P T G D S$ and WNT2B are linked with vasohibin 2

Table 1 Genes differentially DNA methylated and expressed in fibrotic HIF

\begin{tabular}{|c|c|c|c|c|}
\hline Gene symbol & Hyper/hypo & Location of DMR & Fold change (B vs. A) & Adjusted $p$ value \\
\hline WNT2B & 01 & Promoter + 5' UTR + exon + intron & 0.19 & 0 \\
\hline PTGIS & 01 & Promoter + intron & 0.18 & 0 \\
\hline PTGDS $^{a}$ & 10 & Promoter + exon & 6.44 & 0.01 \\
\hline COL24A1 & 01 & Promoter $+5^{\prime}$ flank & 5.94 & 0.01 \\
\hline OBSCN & 01 & Promoter + exon + intron & 3.47 & 0.04 \\
\hline RAB38 & 01 & Promoter $+5^{\prime}$ UTR + exon $+5^{\prime}$ flank & 5.73 & 0 \\
\hline$A R L 4 C$ & 01 & Intergenic + promoter $+5^{\prime}$ flank & 3.43 & 0.04 \\
\hline CLIC6 & 01 & Intergenic + promoter $+5^{\prime}$ flank & 3.64 & 0.02 \\
\hline FBLN1 & 01 & Intergenic + promoter $+5^{\prime}$ Flank & 0.22 & 0 \\
\hline KALRN & 01 & Promoter $+5^{\prime}$ UTR + exon + intron $+5^{\prime}$ flank & 3.64 & 0.03 \\
\hline$D C D C 2$ & 01 & Intergenic + promoter $+5^{\prime}$ flank & 25.14 & 0.01 \\
\hline $\mathrm{KCNH} 2$ & 01 & Promoter + exon + intron & 0.13 & 0 \\
\hline$|F| 27$ & 10 & Promoter $+5^{\prime}$ UTR + exon + intron & 4.06 & 0.04 \\
\hline AOC3 & 10 & Promoter $+5^{\prime}$ UTR + exon + intron & 0.04 & $1.16 \mathrm{E}-15$ \\
\hline ADAMTS5 & 10 & Promoter + intron & 5.11 & 0 \\
\hline
\end{tabular}

The genes that were found to be both differentially DNA methylated in their promoter regions and inversely correlated with gene expression are shown. Hyper/ hypo column denotes whether genes are yes/no hyper- (01) or hypo- (10) methylated in fibrotic HIF. Italicized 01 and 10 represent genes that were quantitatively differentially DNA methylated and not analyzed further in this study. The location of the DMR, the fold change in mRNA expression detected by RNA-seq, and the adjusted $p$ value are shown

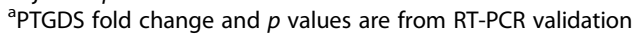




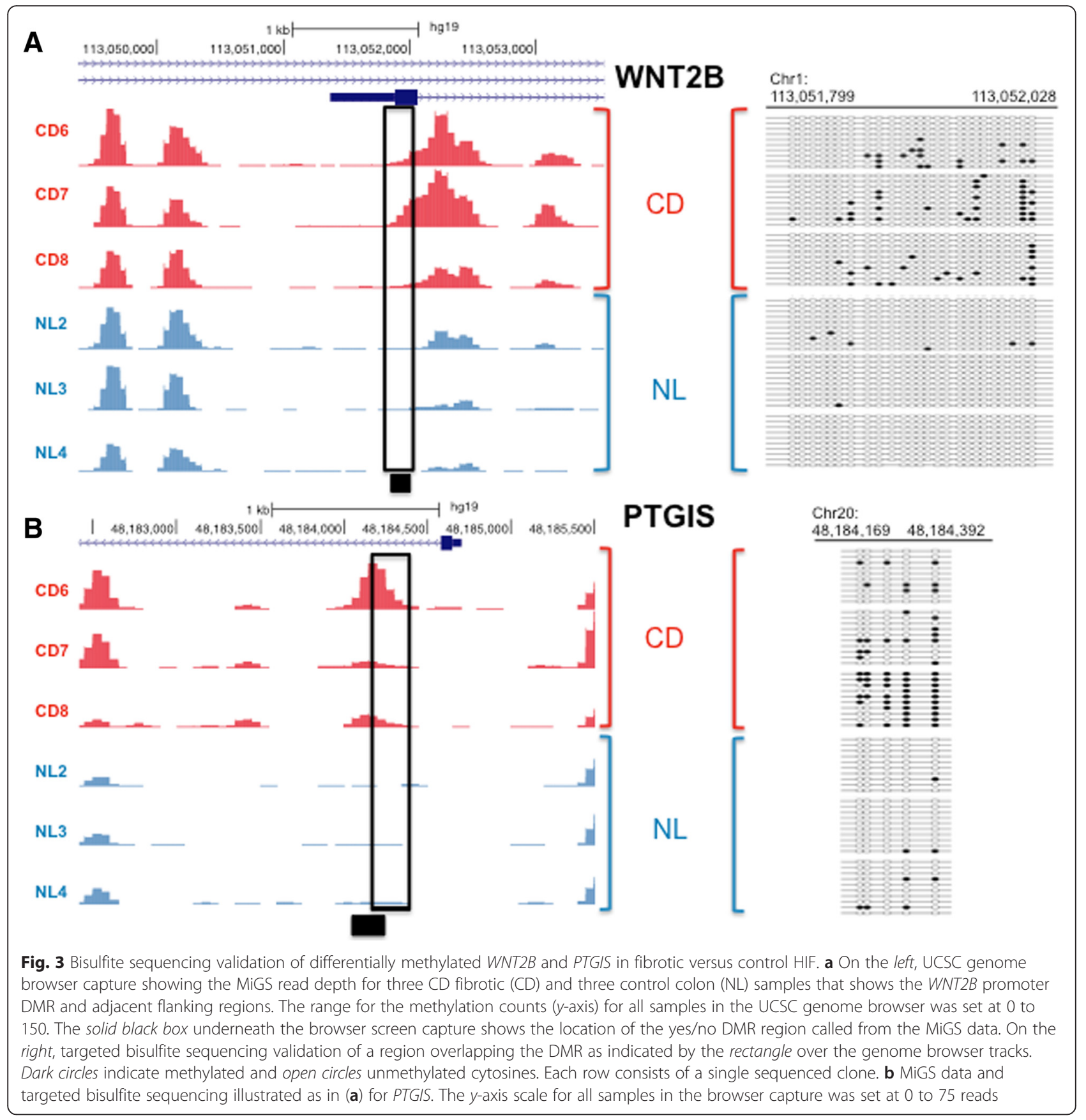

(VASH2) [45] and chromosome 7 open reading frame 69 (C7orf69).

\section{Discussion}

The objective of our studies was to obtain novel insights into the molecular mechanisms that underlie intestinal fibrogenesis. Through definition and integration of the DNA methylome and transcriptome, we have revealed functional candidate gene networks for three proteincoding genes: WNT2B, PTGIS, and PTGDS, which have not previously been described in $\mathrm{CD}$ or other fibrotic diseases. By employing the next generation sequencingbased approach, MiGS [24] and RNA-seq, we were able to achieve genome-wide coverage and improved accuracy over previous studies that have used microarrays to profile changes in DNA methylation and gene expression in inflammatory bowel disease and other fibrotic disorders.

Our experiments were performed in HIF purified from fibrotic or normal colon tissue to minimize the confounding effects of cell type heterogeneity of whole blood or tissue that have been widely used in previous 


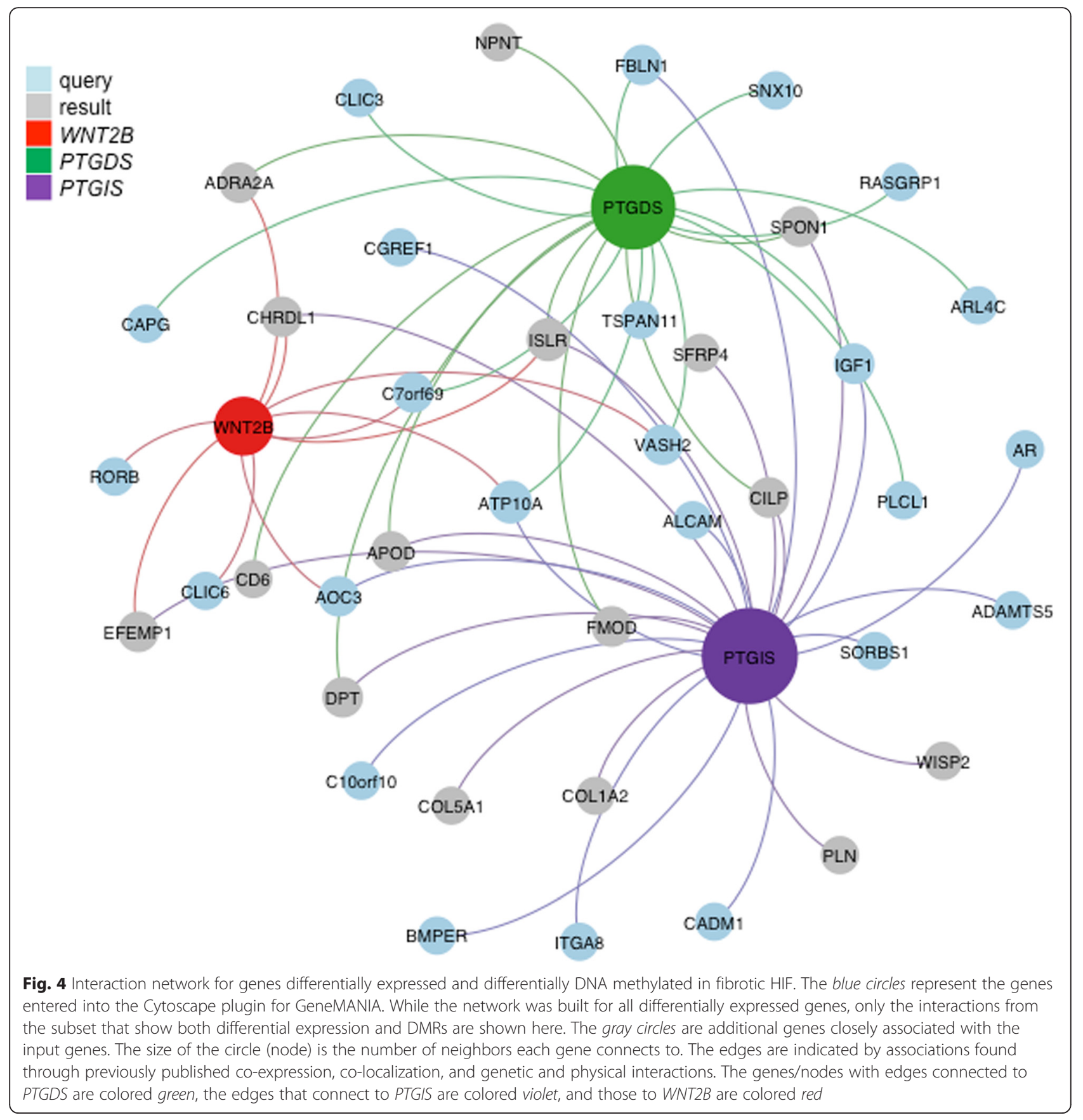

epigenetic studies [28]. The large number, size, and genome-wide distribution of DMRs in fibrotic HIF suggest that this epigenetic modification has an important role in intestinal fibrosis. We observed sharp yes/no differences in DNA methylation throughout the genic and intergenic regions of the fibrotic HIF genome. Only a comparatively low percentage of DMRs occurred in promoters of genes and in CpG islands. The vast majority of DMRs were located within introns and intergenic regions. Intergenic sequences contain enhancers and insulators that are associated with regulation of gene expression during differentiation and organogenesis $[7,8]$. Other distinct functions for DNA methylation have been proposed within introns and $\mathrm{CpG}$ island shores $[28,46,47]$. For example, DNA methylation in introns can modulate alternative exon splicing $[46,47]$ and in shores, differential DNA methylation is tissuespecific and may regulate transcription from alternative start sites [28].

The precise mechanism by which DNA methylation regulates gene transcription remains poorly defined. Methylated CpGs can prevent the binding of some 
transcription factors [7], and other evidence indicates that methylation of DNA can alter nucleosome occupancy and alternative polyadenylation of mRNA during transcription [48-50]. Whether de novo DNA methylation directly represses genes or whether gene silencing precedes or follows methylation is still debated, but the available evidence indicates that this will be dependent in large part on the genomic location of the methylated DNA sequence $[7,8]$. This is a rapidly advancing area with new compelling evidence for causal associations between DNA methylation changes and phenotype [51, 52]. Our detection of DMRs provides a resource of candidate loci for future work to understand the role of DNA methylation in different genomic locations at different stages of pro-fibrotic gene expression.

We identified three genes: WNT2B, PTGIS, and PTGDS in which differential methylation of the DNA in each promoter region was inversely correlated with gene expression in fibrotic HIF. Complex roles for the WNT signaling pathway in the fibrosis of a number of organs including the lung and kidney and in controlling tissue homeostasis, cell proliferation, migration, differentiation, apoptosis, and organogenesis have been reported [53, 54]; however, this family of genes has not previously been associated with intestinal fibrosis. The decreased expression of hypermethylated WNT2B is a novel finding both in the context of fibrosis and $C D$. Interestingly, increased expression of $W N T 2 B$ has been detected in the intestinal mucosa of UC patients [55], suggesting that WNT2B may perform distinct functions in $\mathrm{CD}$ and $\mathrm{UC}$.

In the case of the prostaglandin family, central functions are well-established in homeostasis, inflammation, and other fibrosis disorders [56]. Profibrogenic functions for prostaglandin F2 $\alpha$ and anti-fibrotic effects of prostaglandin $E_{2}$ have been documented in lung fibrosis $[57,58]$. In addition, the product of PTGIS catalysis, $\mathrm{PGI}_{2}$, has been shown to be anti-fibrotic in the lung [56] and also in a prostacyclin receptor null mouse model of cardiac fibrosis [59]. We found that PTGIS was hypermethylated and repressed in fibrotic HIF. Hypermethylation of the PTGIS promoter is a feature of colorectal cancer, but decreased expression of PTGIS has not previously been linked with IBD [60]. A further finding of our studies was that the lipocalin-type PTGDS gene was hypomethylated and its mRNA levels increased in fibrotic HIF. Our observations are supported by studies showing (i) increased levels of lipocalin type PTGDS in colitis, (ii) increased expression in UC patients in parallel with disease activity, and (iii) decreased DSS-induced colitis in L-PTGDS knockout mice [61]. Conflicting data in other studies indicate that in the kidney, $\mathrm{PGD}_{2}$, the product of PGDS catalytic activity, promotes the development of tubulointerstitial fibrosis [62], whereas a protective function of $\mathrm{PGD}_{2}$ has been reported in TNBS colitis [63]. These observations may reflect tissue-specific differences or methodological discrepancies between the aforementioned studies.

Based on previous studies including a recent study of fibrotic liver disease, the average age difference of 20 years between our fibrotic Crohn's disease and normal patient groups is highly unlikely to be a confounding factor in the differential DNA methylation we identified [23]. Comparison of the DNA methylomes of dermal fibroblasts from individuals with a much greater disparity in age $(<23$ years old vs. $>60$ years old $)$ showed a change in DNA methylation levels of $>15 \%$ at only $75 \mathrm{CpG}$ sites, none of which were highly significant $(p<0.001)$ [64]. More importantly, none of these sites were differentially methylated in fibrotic HIF.

Studies in tissues such as the heart, and in diseases such as cancer, indicate that the same epigenetic pathways that are beneficial in organ development are perturbed in the fully developed or diseased tissue. The concept of antagonistic pleiotropy [65] suggests that key pathways are advantageous at early stages of development, e.g., in myofibroblast differentiation and wound healing, but in later life, these same pathways become detrimental as they promote myofibroblast activation and progressive fibrosis $[65,66]$. Thus, the available evidence indicates that alterations in DNA methylation during development could serve as key determinants of the pro-fibrotic phenotype. Together with genetic and environmental factors, DNA methylation could be a major contributor to the progression of injury in $C D$ and to the establishment of intestinal fibrosis. An important aim of future studies will be to determine changes in DNA methylation in patients with different stages of fibrostenotic $\mathrm{CD}$. This could help stratify distinct subtypes of $\mathrm{CD}$ and identify those patients most likely to develop intestinal fibrosis

\section{Conclusions}

Our detailed map of the methylome integrated with the transcriptome has revealed fibrosis-associated regulation of WNT2B, PTGIS, and PTGDS. The networks for these genes reveal interactions with other factors important in development and extracellular matrix synthesis. This suggests that key sites of differential DNA methylation can lead to the molecular aberrations that underlie IBDassociated fibrosis and provide potential targets for future development of prognostic strategies and specific anti-fibrotic therapies. Our data also provide a basis for defining the contribution of genome-wide changes in DNA methylation to the pathogenesis of fibrotic disorders. 


\section{Methods}

\section{Colon tissue and cell culture}

Colon resection specimens were obtained from patients with CD-associated fibrosis and control colon specimens were obtained from histologically normal tissue from patients with diverticulitis (Additional file 8) under the approval of the Cleveland Clinic institutional review board (IRB 06-050). The approved protocol included a waiver of informed consent for redundant tissues obtained through the Cleveland Clinic Human Tissue Procurement Facility in accordance with Cleveland Clinic IRB policy. Tissue specimens were deidentified and made available following authorization by a Cleveland Clinic surgical pathologist. Human intestinal fibroblasts (HIF) were cultured from colon specimens and their purity confirmed as previously reported by established methods [67].

\section{DNA methylome profiling}

Genome-wide DNA methylation was mapped using methyl-CpG binding domain-isolated genome sequencing (MiGS) [24], with the following differences. Genomic DNA (DNEasy kit, Qiagen) was extracted from normal and fibrotic cultured HIF. DNA $(10 \mu \mathrm{g})$ was sheared on a Covaris S220 to an average size of $120 \mathrm{bp}$. Methylated DNA fragments were purified on PrepEase DNA columns (USB) and captured with the methyl-CpG binding protein MBD2 (MethylMiner Methylated DNA Enrichment kit \#ME10025, Invitrogen). A sequencing library for each immunoprecipitated DNA sample was prepared using Ilumina's ChIP-seq DNA Sample Prep Kit. The quality of the DNA was assessed on an Agilent Bioanalyser. Sequencing libraries of methylated DNA fragments were analyzed and next generation sequencing performed on an Ilumina HiSeq 2000. Read lengths of 50 bp were sequenced at an average depth of 161,226,405 reads (minimum: 130,528,176 reads, maximum: 190,943,105 reads). Reads were aligned to assembly $h g 19$ of the human genome (Genome Reference Consortium Human Build 37) using the bowtie 2 short read alignment software (options: -N 1 -L 20 -phred33) [68].

\section{Bioinformatic analysis of differential DNA methylation}

Differential DNA methylation was detected using the integrated signal deconvolution, pattern recognition, and differential testing (iDPT) framework [69] (https://idpt. github.io/dptscan/). Using this framework, reads were extended to the average fragment length. Average coverage (e.g., averages of the per-base coverage rounded to integers) was computed within 50-bp non-overlapping windows tiling the entire genome. Initial filtering removed all windows where no sample had a read count above a false discovery threshold established by a Poison distribution that assumed an even coverage of reads across the genome. Signal deconvolution was performed using a Bayesian mixture of three Poisons model, producing a probability of methylation for each window in each sample. Pattern recognition used these probabilities and a scan statistic to produce regions of consistent methylation status, allowing for small gaps.

Finally, differential testing was performed using a linear mixed-effects model and an output table that contained fold changes, and multiple testing-adjusted $p$ values for each DMR was produced. The output differentiated between sites showing a sharp yes/no methylation difference (defined as all samples in one group are predicted by the model to have no methylation, and all samples in the other group are predicted to be methylated) versus sites that show a quantitative difference in reads (the model predicts all samples across all groups to be methylated, but the read counts show a statistically significant difference). Only the sharp sites were used for subsequent analysis because they are more likely to represent differential DNA methylation rather than other phenomena that can change read counts, such as copy number variation.

The $\mathrm{R}$ package goldmine was used to analyze the genomic context of the detected regions with respect to known genes and features (http://jeffbhasin.github.io/ goldmine). The goldmine functions, getCpgFeatures, getFeatures, and getGenes were used to obtain CpG islands, ENCODE supertracks, and RefSeq gene models from the database of the UCSC Genome Browser [70, 71]. This data was used by the goldmine function to provide detailed annotation of how these datasets relate to the regions.

\section{RNA sequencing}

For RNA sequencing (RNA-seq), RNA was extracted from control and fibrotic HIF using the RNEasy mini kit (Qiagen). A gene sequencing library for each sample (1 $\mu$ g RNA) was prepared using the TruSeq ${ }^{\text {TM }}$ RNA Sample Prep Kit v2. Next generation sequencing was performed on an Illumina HiSeq 2500. After quality assessment, reads were aligned to $h g 19$ using GSNAP [72]. Read lengths of $100 \mathrm{bp}$ were sequenced at an average depth of 53,390,000 reads (minimum 39,470,000 reads, maximum $62,550,000$ reads). Only reads that aligned to a single locus in the $h g 19$ genome assembly were retained for further analysis. Read counts for RefSeq genes were computed using HTSeq-count program [73]. Differential expression analysis was performed on this count table using the negative binomial test provided by the DESeq package [74] in the R statistical computing environment (http://www.R-project.org/). Transcripts with $p$ values less than 0.05 after adjustment by the Benjamini-Hochberg procedure were considered statistically significant. 


\section{Bisulfite sequencing}

For validation of DMRs detected by the genome-wide DNA methylome analysis, genomic DNA (330 ng) was prepared using the DNEAsy kit (Qiagen). Bisulfite conversion of the DNA was performed with the EZ DNA Methylation-Lightning Kit \#D5030 (Zymo). Bisulfiteconverted DNA $(1 \mu \mathrm{l})$ was amplified by PCR with iTaq DNA polymerase (BioRad), and primers were designed using the MethPrimer website (see Additional file 9). DNA was cloned (Topo TA cloning kit, K4575-01, Invitrogen), and the cloned DNA (prepared using PureLink Miniprep kit, Invitrogen) was digested with EcoR1 to verify cloning of the inserted DNA. DNA from at least ten different clones was then sequenced at the Genomics Core of Cleveland Clinic's Lerner Research Institute. DNA methylation of individual CpG sites was analyzed using Quma software (http://quma.cdb.riken.jp/).

\section{Reverse transcriptase PCR (RT-PCR)}

For validation of changes in mRNA levels of differentially DNA methylated genes, total RNA was extracted from fibrotic and normal HIF and reverse transcribed, and complementary DNA (cDNA) was amplified by RTPCR. Five microliters of cDNA was amplified in the presence of $0.125 \mu \mathrm{mol} / \mathrm{L}$ each of the $5^{\prime}$ and $3^{\prime}$ primers (Biosynthesis, Lewisville, TX) and $1 \mathrm{U}$ of Taq DNA polymerase (Roche, Mannheim, Germany). PCR was performed in a DNA thermal cycler using pre-optimized temperatures and times, and the primers (listed in Additional file 9) were used to quantify mRNA levels. Fifteen microliters of the PCR product were subjected to electrophoresis on $1.5 \%$ agarose gel and stained with $0.5 \mu \mathrm{g} / \mathrm{ml}$ ethidium bromide, using $100 \mathrm{bp}$ DNA ladder as a marker.

\section{GeneMANIA network analysis}

The GeneMANIA algorithm (version 3.2.1, http:// www.genemania.org) employs functional interaction data to create a network containing putative functional links between genes. We used the GeneMANIA Cytoscape plugin [75] to generate a network for the genes identified in this study based on protein-protein interactions, genetic interactions, and co-expression profile databases. Data on shared protein domains and co-localization were eliminated from the analysis, to minimize the number of false positives. A sub-network was extracted by restricting the network to only nodes that interact with defined genes of interest and was plotted using Gephi (https://gephi.github.io/).

\section{Availability of supporting data}

The DNA methylation and RNA-seq data sets (raw and normalized) supporting the results of this article are available in the NCBI Gene Expression Omnibus (GEO) repository: http://www.ncbi.nlm.nih.gov/geo/, accession number GSE67250.

\section{Additional files}

Additional file 1: Table S1. Differentially methylated regions (DMRs) between normal and fibrotic HIF. Each row represents one DMR. For each DMR, a unique ID, coordinates in the form of chr, start, end (hg19), and the DMR width are provided. Each DMR is categorized by a pattern code: "01" represents hypermethylation in fibrotic samples, and "10" represents hypomethylation in fibrotic samples. The "ratio" column represents the magnitude of the difference in sequencing signal as a $\log 2$ fold change, "p.value" is the unadjusted $p$ value, and "q.value" is the Benjamini-Hochberg adjusted $p$ value. "Genomic_context" specifies if the DMR falls within a portion of an annotated gene model. Multiple overlaps are resolved using the priority: promoter $>$ exon $>$ intron $>3^{\prime}$ end $>$ intergenic. Overlap with CpG shores, islands, and shelves is provided, along with the distance to the nearest gene and the names of the nearest genes. (XLS $461 \mathrm{~kb})$

Additional file 2: Table S2. RNA-seq analysis of differentially expressed genes in control and fibrotic HIF. Total mRNAs from three different normal $(2,3,4)$ and fibrotic fibroblasts $(6,7,8)$ were subjected to RNA-seq analysis. Normalized FPKM values were averaged and the log base twofold change for fibrotic versus normal HIF calculated. FDR adjusted $p$ values are shown. Genes were considered significant if the adjusted $p$ value was $<0.05$ and the log fold change was at least 1.5 -fold. The differentially expressed genes are highlighted. (XLS $45 \mathrm{~kb}$ )

Additional file 3: Figure S1. Bisulfite sequencing validation of differentially methylated PTGDS in fibrotic versus control HIF. UCSC genome browser capture (left panel) of regions of differential DNA methylation for PTGDS from MiGS are shown next to the corresponding region of the gene validated by bisulfite sequencing (right panel). Three $\mathrm{CD}$ fibrotic (top panel) and three control (NL, lower panel) samples are shown. Dark circles indicate methylated and open circles unmethylated cytosines. Each row consists of a single sequenced clone. The range for the methylation counts ( $y$-axis) in the UCSC genome browser was set at 1 to 128 . (TIFF $1517 \mathrm{~kb}$ )

Additional file 4: Table S3. List of input genes for GeneMANIA. The list of genes used to construct an interaction network in GeneMANIA in order to obtain functional enrichment is provided. A subset of the identified interactions are shown in Fig. 4. (XLSX 9 kb)

Additional file 5: Table S4. Network gene associations for WNT2B. List of genes in RNA-seq data set (bold italics) and in GeneMANIA database (italics) that interact with WNT2B are shown. (XLSX $45 \mathrm{~kb}$ )

Additional file 6:Table S5. Network gene associations for PTGIS. List of genes in RNA-seq data set (bold italics) and in GeneMANIA database (italics) that interact with PTG/S are shown. (XLSX $45 \mathrm{~kb}$ )

Additional file 7:Table S6. Network gene associations for PTGDS. List of genes in RNA-seq data set (bold italics) and in GeneMANIA database (italics) that interact with PTGDS are shown. (XLSX $41 \mathrm{~kb}$ )

Additional file 8: Table S7. Patient characteristics. The characteristics of the patients with Crohn's disease and controls from whom colon specimens were obtained for the DNA methylome and RNA Seq analyses are shown. (XLSX 9 kb)

Additional file 9: Table S8. Primers for gene expression and bisulfite sequencing. The bisulfite sequencing primers for specific chromosomal locations and the GRT-PCR primer sequences and accession numbers, for WNT2B, PTGIS, and PTGDS are shown. (XLSX $11 \mathrm{~kb}$ )

\section{Abbreviations}

CD: Crohn's disease; CGIs: CpG islands; DMRs: differentially methylated regions; ECM: extracellular matrix; FBLN1: fibulin-1; FDR: false discovery rate; HIF: human intestinal fibroblasts; IBD: inflammatory bowel disease; IGF-1: insulin-like growth factor 1; MBD: methyl-CpG binding domain; MiGS: MBD-isolated genome sequencing; PTGDS: prostaglandin D2 synthase; PTGIS: prostacyclin synthase; UC: ulcerative colitis; VASH2: vasohibin 2; 
WNT2B: wingless-type mouse mammary tumor virus integration site family, member $2 \mathrm{~B}$.

\section{Competing interests}

The authors declare that they have no competing interests.

\section{Authors' contributions}

TS performed the experiments and generated and analyzed the data. JMB assembled, analyzed, and interpreted the data. YX and AHT developed the DNA methylome algorithm. YX, JB-S, and YC analyzed and interpreted the data and performed the statistical analysis. TS, JMB, AHT, and ES drafted the manuscript. ES devised the study concept. AHT and ES co-designed the study. ES obtained funding and supervised the study. All authors critically reviewed and approved the final version of the manuscript

\section{Acknowledgements}

Support for TS and ES was from the Broad Foundation (IBD-032) and the National Institutes of Health (NIH) (DK093630 and DK050984). JMB is a predoctoral student in the Molecular Medicine PhD Program of the Cleveland Clinic and Case Western Reserve University that is funded in part by the "Med into Grad" initiative of the Howard Hughes Medical Institute (HHMI). JMB and AHT are supported by the National Cancer Institute, NIH (R01CA154356 and F31CA195887). We are grateful to Dr. Claudio Fiocchi and Gail West, Department of Pathobiology of the Cleveland Clinic and to Eboni Ubinas and Tiffany Hollo and all staff of the Tissue Procurement Service of the Cleveland Clinic for their help with the collection of intestinal tissue specimens. We also gratefully acknowledge the technical assistance with next generation sequencing of the McGill University and Génome Québec Innovation Center, Montreal, Canada, and the Genomics Core, Case Western Reserve University, Cleveland, Ohio.

\section{Author details}

${ }^{1}$ Department of Pathobiology, Cleveland Clinic Lerner Research Institute, 9500 Euclid Avenue/NC-22, Cleveland, OH 44195, USA. ²Department of Molecular Medicine, Cleveland Clinic Lerner College of Medicine at Case Western Reserve University, Cleveland, OH, USA. ${ }^{3}$ Genomic Medicine Institute, Lerner Research Institute, Cleveland Clinic, 9500 Euclid Avenue/NC-22, Cleveland, OH 44195, USA. ${ }^{4}$ Department of Biostatistics, Vanderbilt University School of Medicine, Nashville, TN, USA. Institute for Computational Biology, Case Western Reserve University, Cleveland, OH, USA. ${ }^{6}$ Department of Gastroenterology and Hepatology, Digestive Diseases Institute, Cleveland Clinic, Cleveland, $\mathrm{OH}$, USA.

\section{Received: 7 October 2015 Accepted: 29 February 2016 Published online: 12 March 2016}

\section{References}

1. Kaser A, Zeissig S, Blumberg RS. Inflammatory bowel disease. Annu Rev Immunol. 2010;28:573-621.

2. Ahmed T, Rieder F, Fiocchi C, Achkar JP. Pathogenesis of postoperative recurrence in Crohn's disease. Gut. 2011;60(4):553-62.

3. Strober W. Impact of the gut microbiome on mucosal inflammation. Trends Immunol. 2013;34(9):423-30.

4. Scarpa M, Stylianou E. Epigenetics: concepts and relevance to IBD pathogenesis. Inflamm Bowel Dis. 2012;18(10):1982-96.

5. Ventham NT, Kennedy NA, Nimmo ER, Satsangi J. Beyond gene discovery in inflammatory bowel disease: the emerging role of epigenetics. Gastroenterology. 2013;145(2):293-308.

6. Stylianou E. Epigenetics: the fine-tuner in inflammatory bowel disease? Curr Opin Gastroenterol. 2013;29(4):370-7.

7. Jones PA. Functions of DNA methylation: islands, start sites, gene bodies and beyond. Nat Rev Genet. 2012;13(7):484-92.

8. Schubeler D. Function and information content of DNA methylation. Nature. 2015;517(7534):321-6.

9. Nimmo ER, Prendergast JG, Aldhous MC, Kennedy NA, Henderson P, Drummond HE, et al. Genome-wide methylation profiling in Crohn's disease identifies altered epigenetic regulation of key host defense mechanisms including the Th17 pathway. Inflamm Bowel Dis. 2012;18(5):889-99.

10. Lin Z, Hegarty JP, Yu W, Cappel JA, Chen X, Faber PW, et al. Identification of disease-associated DNA methylation in B cells from Crohn's disease and ulcerative colitis patients. Dig Dis Sci. 2012;57(12):3145-53.
11. Cooke J, Zhang H, Greger L, Silva AL, Massey D, Dawson C, et al. Mucosal genome-wide methylation changes in inflammatory bowel disease. Inflamm Bowel Dis. 2012;18(11):2128-37.

12. Hasler R, Feng Z, Backdahl L, Spehlmann ME, Franke A, Teschendorff A, et al. A functional methylome map of ulcerative colitis. Genome Res. 2012;22(11): 2130-7.

13. Adams AT, Kennedy NA, Hansen R, Ventham NT, O'Leary KR, Drummond HE, et al. Two-stage genome-wide methylation profiling in childhood-onset Crohn's Disease implicates epigenetic alterations at the VMP1/MIR21 and HLA loci. Inflamm Bowel Dis. 2014;20(10):1784-93.

14. Sadler T, Scarpa M, Rieder F, West G, Stylianou E. Cytokine-induced chromatin modifications of the type I collagen alpha 2 gene during intestinal endothelial-to-mesenchymal transition. Inflamm Bowel Dis. 2013;19(7):1354-64.

15. Chen Y, Ge W, Xu L, Qu C, Zhu M, Zhang W, et al. miR-200b is involved in intestinal fibrosis of Crohn's disease. Int J Mol Med. 2012;29(4):601-6.

16. Nijhuis A, Biancheri $P$, Lewis $A$, Bishop CL, Giuffrida $P$, Chan $C$, et al. In Crohn's disease fibrosis-reduced expression of the miR-29 family enhances collagen expression in intestinal fibroblasts. Clin Sci (Lond). 2014;127(5):341-50

17. Wing MR, Devaney JM, Joffe MM, Xie D, Feldman HI, Dominic EA, et al. DNA methylation profile associated with rapid decline in kidney function: findings from the CRIC study. Nephrol Dial Transplant. 2014;29(4):864-72.

18. Ko YA, Mohtat D, Suzuki M, Park AS, Izquierdo MC, Han SY, et al. Cytosine methylation changes in enhancer regions of core pro-fibrotic genes characterize kidney fibrosis development. Genome Biol. 2013;14(10):R108.

19. Komatsu Y, Waku T, Iwasaki N, Ono W, Yamaguchi C, Yanagisawa J. Global analysis of DNA methylation in early-stage liver fibrosis. BMC Med Genomics. 2012;5:5.

20. Huang SK, Scruggs AM, McEachin RC, White ES, Peters-Golden M. Lung fibroblasts from patients with idiopathic pulmonary fibrosis exhibit genomewide differences in DNA methylation compared to fibroblasts from nonfibrotic lung. PLoS One. 2014;9(9):e107055.

21. Rabinovich El, Kapetanaki MG, Steinfeld I, Gibson KF, Pandit KV, Yu G, et al. Global methylation patterns in idiopathic pulmonary fibrosis. PLoS One. 2012;7(4):e33770.

22. Sanders YY, Ambalavanan N, Halloran B, Zhang X, Liu H, Crossman DK, et al. Altered DNA methylation profile in idiopathic pulmonary fibrosis. Am J Respir Crit Care Med. 2012;186(6):525-35.

23. Zeybel M, Hardy T, Robinson SM, Fox C, Anstee QM, Ness T, et al. Differential DNA methylation of genes involved in fibrosis progression in non-alcoholic fatty liver disease and alcoholic liver disease. Clinical epigenetics. 2015;7(1):25

24. Serre $\mathrm{D}$, Lee $\mathrm{BH}$, Ting $\mathrm{AH}$. MBD-isolated genome sequencing provides a high-throughput and comprehensive survey of DNA methylation in the human genome. Nucleic Acids Res. 2010;38(2):391-9.

25. Robinson MD, Strbenac D, Stirzaker C, Statham AL, Song J, Speed TP, et al Copy-number-aware differential analysis of quantitative DNA sequencing data. Genome Res. 2012;22(12):2489-96.

26. Yang X, Han H, De Carvalho DD, Lay FD, Jones PA, Liang G. Gene body methylation can alter gene expression and is a therapeutic target in cancer. Cancer Cell. 2014;26(4):577-90.

27. Feinberg AP. Phenotypic plasticity and the epigenetics of human disease. Nature. 2007:447(7143):433-40.

28. Irizarry RA, Ladd-Acosta C, Wen B, Wu Z, Montano C, Onyango P, et al. The human colon cancer methylome shows similar hypo- and hypermethylation at conserved tissue-specific CpG island shores. Nat Genet. 2009;41(2):178-86.

29. Franchimont D, Vermeire S, El Housni H, Pierik M, Van Steen K, Gustot T, et al. Deficient host-bacteria interactions in inflammatory bowel disease? The toll-like receptor (TLR)-4 Asp299gly polymorphism is associated with Crohn's disease and ulcerative colitis. Gut. 2004;53(7):987-92.

30. Pastorelli L, De Salvo C, Cominelli MA, Vecchi M, Pizarro TT. Novel cytokine signaling pathways in inflammatory bowel disease: insight into the dichotomous functions of IL-33 during chronic intestinal inflammation. Therap Adv Gastroenterol. 2011;4(5):311-23.

31. Simmons JG, Pucilowska JB, Keku TO, Lund PK. IGF-I and TGF-beta1 have distinct effects on phenotype and proliferation of intestinal fibroblasts. Am J Physiol Gastrointest Liver Physiol. 2002;283(3):G809-18.

32. McHedlidze T, Waldner M, Zopf S, Walker J, Rankin AL, Schuchmann M, et al. Interleukin-33-dependent innate lymphoid cells mediate hepatic fibrosis. Immunity. 2013;39(2):357-71. 
33. Seki E, De Minicis S, Osterreicher CH, Kluwe J, Osawa Y, Brenner DA, et al. TLR4 enhances TGF-beta signaling and hepatic fibrosis. Nat Med. 2007;13(11):1324-32.

34. Poulain M, Ober EA. Interplay between Wnt2 and Wnt2bb controls multiple steps of early foregut-derived organ development. Development. 2011;138(16):3557-68.

35. Yokoyama C, Yabuki T, Inoue H, Tone Y, Hara S, Hatae T, et al. Human gene encoding prostacyclin synthase (PTGIS): genomic organization, chromosomal localization, and promoter activity. Genomics. 1996;36(2):296304.

36. Joo M, Sadikot RT. PGD synthase and PGD2 in immune resposne. Mediators Inflamm. 2012;2012:503128.

37. Rieder F, Fiocchi C. Intestinal fibrosis in IBD—a dynamic, multifactorial process. Nat Rev Gastroenterol Hepatol. 2009;6(4):228-35.

38. Spagnolo P, Sverzellati N, Rossi G, Cavazza A, Tzouvelekis A, Crestani B, Vancheri C. Idiopathic pulmonary fibrosis: an update. Ann Med. 2015;47(1): $15-27$.

39. Narumiya S. The small GTPase Rho: cellular functions and signal transduction. J Biochem. 1996;120(2):215-28.

40. Choi SS, Sicklick JK, Ma Q, Yang L, Huang J, Qi Y, et al. Sustained activation of Rac1 in hepatic stellate cells promotes liver injury and fibrosis in mice. Hepatology. 2006;44(5):1267-77.

41. Tarnawski AS. Cellular and molecular mechanisms of gastrointestinal ulcer healing. Dig Dis Sci. 2005;50 Suppl 1:S24-33.

42. Jaffar J, Unger S, Corte TJ, Keller M, Wolters PJ, Richeldi L, et al. Fibulin-1 predicts disease progression in patients with idiopathic pulmonary fibrosis. Chest. 2014;146(4):1055-63.

43. Hattori N, Carrino DA, Lauer ME, Vasanji A, Wylie JD, Nelson CM, et al. Pericellular versican regulates the fibroblast-myofibroblast transition: a role for ADAMTS5 protease-mediated proteolysis. J Biol Chem. 2011;286(39): 34298-310.

44. Schnapp LM, Hatch N, Ramos DM, Klimanskaya IV, Sheppard D, Pytela R. The human integrin alpha 8 beta 1 functions as a receptor for tenascin, fibronectin, and vitronectin. J Biol Chem. 1995:270(39):23196-202.

45. Wang $X$, Zhu H, Yang X, Bi Y, Cui S. Vasohibin attenuates bleomycin induced pulmonary fibrosis via inhibition of angiogenesis in mice. Pathology. 2010;42(5):457-62.

46. Maunakea AK, Nagarajan RP, Bilenky M, Ballinger TJ, D'Souza C, Fouse SD, et al. Conserved role of intragenic DNA methylation in regulating alternative promoters. Nature. 2010:466(7303):253-7.

47. Shukla S, Kavak E, Gregory M, Imashimizu M, Shutinoski B, Kashlev M, et al. CTCF-promoted RNA polymerase II pausing links DNA methylation to splicing. Nature. 2011;479(7371):74-9.

48. Choy JS, Wei S, Lee JY, Tan S, Chu S, Lee TH. DNA methylation increases nucleosome compaction and rigidity. J Am Chem Soc. 2010;132(6):1782-3.

49. Wood AJ, Schulz R, Woodfine K, Koltowska K, Beechey CV, Peters J, et al. Regulation of alternative polyadenylation by genomic imprinting. Genes Dev. 2008;22(9):1141-6.

50. Lay FD, Triche Jr TJ, Tsai YC, Su SF, Martin SE, Daneshmand S, et al. Reprogramming of the human intestinal epigenome by surgical tissue transposition. Genome Res. 2014:24(4):545-53.

51. Yu DH, Waterland RA, Zhang P, Schady D, Chen MH, Guan Y, et al. Targeted p16(Ink4a) epimutation causes tumorigenesis and reduces survival in mice. J Clin Invest. 2014;124(9):3708-12.

52. Chaikind B, Ostermeier M. Directed evolution of improved zinc finger methyltransferases. PLoS One. 2014;9(5):e96931.

53. Kahn M. Can we safely target the WNT pathway? Nat Rev Drug Discov. 2014;13(7):513-32

54. Verzi MP, Shivdasani RA. Wnt signaling in gut organogenesis. Organogenesis. 2008;4(2):87-91.

55. You J, Nguyen AV, Albers CG, Lin F, Holcombe RF. Wnt pathway-related gene expression in inflammatory bowel disease. Dig Dis Sci. 2008;53(4):1013-9.

56. Castelino FV. Lipids and eicosanoids in fibrosis: emerging targets for therapy. Curr Opin Rheumatol. 2012;24(6):649-55.

57. Wilborn J, Crofford LJ, Burdick MD, Kunkel SL, Strieter RM, Peters-Golden M. Cultured lung fibroblasts isolated from patients with idiopathic pulmonary fibrosis have a diminished capacity to synthesize prostaglandin E2 and to express cyclooxygenase-2. J Clin Invest. 1995;95(4):1861-8.

58. Oga T, Matsuoka T, Yao C, Nonomura K, Kitaoka S, Sakata D, et al. Prostaglandin F(2alpha) receptor signaling facilitates bleomycin-induced pulmonary fibrosis independently of transforming growth factor-beta. Nat Med. 2009:15(12):1426-30.

59. Francois H, Athirakul K, Howell D, Dash R, Mao L, Kim HS, et al. Prostacyclin protects against elevated blood pressure and cardiac fibrosis. Cell Metab. 2005;2(3):201-7.

60. Frigola J, Munoz M, Clark SJ, Moreno V, Capella G, Peinado MA. Hypermethylation of the prostacyclin synthase (PTGIS) promoter is a frequent event in colorectal cancer and associated with aneuploidy. Oncogene. 2005;24(49):7320-6.

61. Hokari R, Kurihara C, Nagata N, Aritake K, Okada Y, Watanabe C, et al. Increased expression of lipocalin-type-prostaglandin D synthase in ulcerative colitis and exacerbating role in murine colitis. Am J Physiol Gastrointest Liver Physiol. 2011;300(3):G401-8.

62. Ito H, Yan X, Nagata N, Aritake K, Katsumata Y, Matsuhashi T, et al. PGD2CRTH2 pathway promotes tubulointerstitial fibrosis. J Am Soc Nephrol. 2012;23(11):1797-809.

63. Ajuebor MN, Singh A, Wallace JL. Cyclooxygenase-2-derived prostaglandin $D(2)$ is an early anti-inflammatory signal in experimental colitis. Am J Physiol Gastrointest Liver Physiol. 2000;279(1):G238-44.

64. Koch CM, Suschek CV, Lin Q, Bork S, Goergens M, Joussen S, et al. Specific age-associated DNA methylation changes in human dermal fibroblasts. PLoS One. 2011;6(2):e16679.

65. Thannickal VJ. Aging, antagonistic pleiotropy and fibrotic disease. Int J Biochem Cell Biol. 2010:42(9):1398-400.

66. Hecker L, Vittal R, Jones T, Jagirdar R, Luckhardt TR, Horowitz JC, et al. NADPH oxidase-4 mediates myofibroblast activation and fibrogenic responses to lung injury. Nat Med. 2009;15(9):1077-81.

67. Strong SA, Pizarro TT, Klein JS, Cominelli F, Fiocchi C. Proinflammatory cytokines differentially modulate their own expression in human intestinal mucosal mesenchymal cells. Gastroenterology. 1998;114(6):1244-56.

68. Langmead B, Salzberg SL. Fast gapped-read alignment with Bowtie 2. Nat Methods. 2012;9(4):357-9.

69. Xu Y, Hu B, Choi AJ, Gopalan B, Lee BH, Kalady MF, et al. Unique DNA methylome profiles in $\mathrm{CpG}$ island methylator phenotype colon cancers. Genome Res. 2012;22(2):283-91.

70. Karolchik D, Barber GP, Casper J, Clawson H, Cline MS, Diekhans M, et al. The UCSC Genome Browser database: 2014 update. Nucleic Acids Res. 2014;42(Database issue):D764-70.

71. Rosenbloom KR, Sloan CA, Malladi VS, Dreszer TR, Learned K, Kirkup VM, et al. ENCODE data in the UCSC Genome Browser: year 5 update. Nucleic Acids Res. 2013;41(Database issue):D56-63.

72. Wu TD, Nacu S. Fast and SNP-tolerant detection of complex variants and splicing in short reads. Bioinformatics. 2010;26(7):873-81.

73. Anders S, Pyl PT, Huber W. HTSeq - a python framework to work with high-throughput sequencing data. Bioinformatics. 2015;31(2):166-9.

74. Anders S, Huber W. Differential expression analysis for sequence count data. Genome Biol. 2010;11(10):R106.

75. Montojo J, Zuberi K, Rodriguez H, Kazi F, Wright G, Donaldson SL, et al. GeneMANIA Cytoscape plugin: fast gene function predictions on the desktop. Bioinformatics. 2010;26(22):2927-8.

\section{Submit your next manuscript to BioMed Central and we will help you at every step:}

- We accept pre-submission inquiries

- Our selector tool helps you to find the most relevant journal

- We provide round the clock customer support

- Convenient online submission

- Thorough peer review

- Inclusion in PubMed and all major indexing services

- Maximum visibility for your research

Submit your manuscript at www.biomedcentral.com/submit 